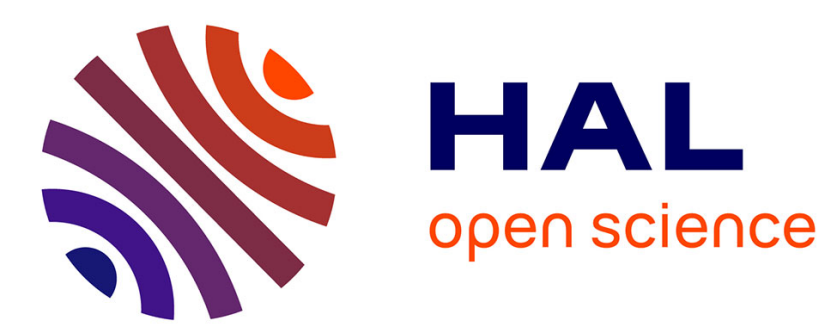

\title{
THE EFFECT OF INITIAL PRESSURE ON TWO AND THREE DIMENSIONAL SOLIDS
}

\author{
G. Stewart
}

\section{To cite this version:}

G. Stewart. THE EFFECT OF INITIAL PRESSURE ON TWO AND THREE DIMENSIONAL SOLIDS. Journal de Physique Colloques, 1977, 38 (C4), pp.C4-207-C4-213. 10.1051/jphyscol:1977431 . jpa-00217147

\section{HAL Id: jpa-00217147 https://hal.science/jpa-00217147}

Submitted on 1 Jan 1977

HAL is a multi-disciplinary open access archive for the deposit and dissemination of scientific research documents, whether they are published or not. The documents may come from teaching and research institutions in France or abroad, or from public or private research centers.
L'archive ouverte pluridisciplinaire HAL, est destinée au dépôt et à la diffusion de documents scientifiques de niveau recherche, publiés ou non, émanant des établissements d'enseignement et de recherche français ou étrangers, des laboratoires publics ou privés. 


\title{
THE EFFECT OF INITIAL PRESSURE ON TWO AND THREE DIMENSIONAL SOLIDS
}

\author{
G. A. STEWART \\ Department of Physics University of Pittsburgh, \\ Pittsburgh, PA 15260, U.S.A.
}

\begin{abstract}
Résumé. - On examine les propriétés élastiques des solides de Cauchy à deux et trois dimensions lorsqu'ils sont soumis à une pression initiale. On montre que leur détermination peut être faite en utilisant uniquement les isothermes. Dans les systèmes à deux et trois dimensions, les vitesses du son, le module de Poisson et la température de Debye dépendent d'un paramètre sans dimension qui est le produit pression-compressibilité. On montre que ce produit possède une valeur maximum de 0,5 indépendante de la dimensionnalité du système. Ce maximum correspond à l'annulation du module de cisaillement et la disparition simultanée des modes sonores transmis. Pour une certaine valeur du produit pression-compressibilité, l'effet de la pression initiale sur les propriétés élastiques ainsi déduites est toujours plus important à deux dimensions qu'à trois. On compare le modèle aux résultats expérimentaux obtenus avec l'hélium et le néon dans le cas tridimensionnel, avec l'hélium adsorbé sur graphite dans le cas bidimensionnel.
\end{abstract}

\begin{abstract}
The elastic properties of two and three-dimensional Cauchy solids subject to initial pressure are examined and shown to require only experimental isotherm information for application. In two and three dimensions the sound speeds, Poisson's ratio, and the Debye temperatures depend upon a dimensionless parameter given by the product of pressure and compressibility. It is shown that the pressure-compressibility product has a maximum value of 0.5 , independent of dimensionality, and that this maximum corresponds to a vanishing shear modulus and the concommitant disappearance of transverse sound modes. For a given value of the pressure-compressibility product, the effect of initial pressure on the elastically derived properties is always greater in two dimensions than in three. The model is compared to direct experimental information for bulk helium and neon, and to the two-dimensional solid case of helium adsorbed on graphite.
\end{abstract}

1. Introduction. - Attempts to describe the physical properties of adsorbed phases in monolayer films on homogeneous solid surfaces lead quickly to considerations of both lower dimensionality and substrate surface structure. The present comments are concerned with one aspect of the former : the role of dimensionality in elastic continuum models intended for application to physisorbed monolayer solids. In particular, we shall be concerned with constructing an elastic model for solids that (i) requires for its utility the minimal mechanical information contained in isotherms and (ii) which may be related to alternative thermal probes like heat capacity measurements.

Noble gases adsorbed on graphite substrates are well established as systems which reveal numerous surface phases including some that exhibit solidlike behaviour. The apparent similarity in certain cases to corresponding phases in the bulk is also well known. In the case of helium, for example, at the higher densities but still below monolayer coverage, heat capacity experiments [1] indicate solids which (a) have Debye temperatures that are essentially the same as in the bulk counterpart phases at the same interatomic separation and which (b) have melting temperatures which vary with interatomic separation much as in the corresponding bulk system. For the heavier noble gases adsorbed on graphite, a number of monolayer fluid and solid phases have also been inferred by precision vapour pressure isotherm experiments $[2,3,4]$ and, more recently, diffraction studies [5]. Some of the proposed phase diagrams for these heavy gas systems also have qualitative similarities to their bulk counterparts with suggested homogeneous solid, liquid, and vapour regions as well as phase co-existence. These similarities in monolayer and bulk systems suggest model comparisons where the same simplifications and assumptions are made in two and three-dimensional treatments. Such a dimensionally isomorphic approach should at least make explicit the influence of dimensionality and, to the extent that substrate influences are not the dominant feature of planar behaviour, may lead to reasonable quantitative agreement with a variety of surface experiments. 
The absence of detailed experimental information on the elastic properties of solid monolayers limits the complexity of theoretical models if they are to be based upon experimental input. As a consequence, models may be required that depend only on the compressibility $K_{2 T}$ and spreading pressure $\phi$ via differentiation and integration of the adsorption isotherm with respect to pressure :

$$
\begin{aligned}
K_{2 T} & =\frac{A}{n_{T}^{2} v_{\mathrm{g}}} \frac{\partial n_{T}(p)}{\partial p} \\
\phi & =\frac{1}{A} \int v_{\mathrm{g}}(p) n_{T}(p) \mathrm{d} p .
\end{aligned}
$$

In (1) and (2), $K_{2 T}=-A^{-1}(\partial A / \partial \phi)_{T}$ is the two-dimensional isothermal compressibility, $v_{\mathrm{g}}(p)$ is the atomic volume of the bulk vapor, $A$ is the total area of the monolayer and $n_{T}(p)$ is the adsorption isotherm. Our specific question for adsorption systems is the following: what two-dimensional model solid can be constructed using all the isotherm information contained in (1) and (2) but which requires no additional elastic information? The corresponding model may be applied for comparison purposes to three dimensional systems where the relevant experimental information is obtainable from bulk equation of state studies.

The general stress-strain relation for a two-dimensional isotropic solid is of the form

$\left(\begin{array}{l}T_{11} \\ T_{22} \\ T_{12}\end{array}\right)=\left(\begin{array}{ccc}\lambda_{2}+2 \mu_{2} & \lambda_{2} & 0 \\ \lambda_{2} & \lambda_{2}+2 \mu_{2} & 0 \\ 0 & 0 & \mu_{2}\end{array}\right)\left(\begin{array}{l}e_{11} \\ e_{22} \\ e_{12}\end{array}\right)$

where the elastic constants in (3) have been chosen as the two-dimensional Lame' constants $\lambda_{2}$ and $\mu_{2}$ (shear modulus) with dimensions of two-dimensional spreading pressure $\phi$. The $e_{i j}$ represent small strains from the initial state which for present purposes we regard as unstressed. Since the general isotropic solid has two independent elastic constants, a model based on the compressibility from (1) is isotropic $a$ fortioni.

2. Cauchy solids in two and three dimensions. Further simplification of (3) is possible for specific symmetries and potentials. If it is assumed, for example, that (i) each atom is located at a center of symmetry and (ii) the atoms interact only through central forces, then the Cauchy conditions $[6,7]$ may be applied. For this situation $\lambda=\mu$ and the stress-strain relation (3) involves only a single independent elastic variable. Equivalently, for this special case, the compressibility contains all the elastic information required to describe the properties of the solid as may be seen by generating $K_{2 T}$ from (3). Regarding the two-dimensional compressibility as the ratio of fractional change in area to applied spreading pressure under uniform hydrostatic compression in the plane,
$T_{11}=T_{22}=-\phi, e_{11}=e_{22}=e, T_{12}=0$ and from (3) the compressibility $K_{2 T}$ is given by [8]

$$
\begin{aligned}
K_{2 T} & \equiv-\frac{1}{A}\left(\frac{\partial A}{\partial \phi}\right)_{T}=-\frac{2 e}{T} \\
& =\left(\lambda_{2}+\mu_{2}\right)^{-1}
\end{aligned}
$$

which simplifies to

$$
K_{2 T}=\left(2 \lambda_{2}\right)^{-1}=(2 \mu)^{-1}
$$

if the Cauchy conditions are assumed. It is apparent that a Cauchy solid without initial stress is a model arising from well defined assumptions and requires only the pressure derivative (1) of the adsorption isotherm for complete specification. For comparison purposes with bulk materials, assuming an isotropic solid characterized by $\lambda_{3}$ and $\mu_{3}$, the stress-strain relation (3) would be $6 \times 6$ and yield the usual

$$
\begin{aligned}
K_{3 T} & =-\frac{1}{V}\left(\frac{\partial V}{\partial p}\right)_{T} \\
& =\left(\lambda_{3}+\frac{2}{3} \mu_{3}\right)^{-1}
\end{aligned}
$$

which simplifies to the Cauchy result for bulk materials

$$
K_{3 T}=\left(\frac{5}{3} \lambda_{3}\right)^{-1}=\left(\frac{5}{3} \mu_{3}\right)^{-1}
$$

when $\lambda_{3}=\mu_{3}$.

Comparison of (4) and (6) indicates that with a non-zero shear modulus, the form of the compressibility as a function of the Lame' elastic constants depends on dimensionality. It is also of interest to examine Poisson's ratio $\sigma$ for effects of dimensionality since, in either two or three dimensions, $\sigma$ is a dimensionless ratio of lateral fractional contraction (strain) to fractional longitudinal contraction under longitudinal stress with free lateral perimeters. The dimensionless character of Poisson's ratio makes it useful for comparison of two and three-dimensional solids subject to the same (Cauchy) assumptions. Manipulation of the stress-strain relation for this particular deformation which defines Poisson's ratio yields in two and three dimensions

$$
\begin{aligned}
\sigma_{2} & =-\frac{e_{22}}{e_{11}} \\
& =\lambda_{2}\left(\lambda_{2}+2 \mu_{2}\right)^{-1} \\
\sigma_{3} & =-\frac{e_{22}}{e_{11}}=-\frac{e_{33}}{e_{11}} \\
& =\frac{\lambda_{3}}{2}\left(\lambda_{3}+\mu_{3}\right)^{-1} .
\end{aligned}
$$

As with the compressibility, the form for Poisson's ratio as a function of the Lame' constants depends 
on dimensionality. Further, we note that the limiting values when the shear modulus vanishes are

$$
\sigma_{2}\left(\mu_{2}=0\right)=1
$$

and

$$
\sigma_{3}\left(\mu_{3}=0\right)=\frac{1}{2}
$$

as expected for fluidlike behaviour when area and volume are conserved in two or three dimensions, respectively. The Cauchy values of $\sigma_{2}$ and $\sigma_{3}$ for $\lambda=\mu$ are given by

$$
\begin{aligned}
& \sigma_{2}(\text { Cauchy })=\frac{1}{3} \\
& \sigma_{3}(\text { Cauchy })=\frac{1}{4}
\end{aligned}
$$

and again show the effects of dimensionality on the elastic properties of a Cauchy solid.

It may be noted that the comparative elastic properties of the two and three-dimensional solids described so far have been a consequence of the familiar form (3) for the stress-strain relation which has implicitly assumed zero initial stress on the system. However, if the Cauchy conditions are to be applied to a solid in an initial state which is stressed then a more general stress-strain relation is required where the elastic Lame' constants are defined with respect to that initial state [11]. A particularly simple case of initial stress is the situation where there exists an initial hydrostatic pressure $p_{0}$ or $\phi_{0}$. In the case of solid helium, for example, the solid always exists under an initial stress. Denoting by $\delta T_{\text {if }}$ the excess stress with respect to an initially hydrostatically stressed state, the requisite generalization of $(3)$ is

$$
\begin{aligned}
\left(\begin{array}{l}
\delta T_{11} \\
\delta T_{22} \\
\delta T_{13}
\end{array}\right) & =\left(\begin{array}{ccc}
\lambda^{\prime}\left(\phi_{0}\right)+2 \mu^{\prime}\left(\phi_{0}\right) & \lambda^{\prime}\left(\phi_{0}\right) & 0 \\
\lambda^{\prime}\left(\phi_{0}\right) & \lambda^{\prime}\left(\phi_{0}\right)+2 \mu^{\prime}\left(\phi_{0}\right) & 0 \\
0 & 0 & \mu^{\prime}\left(\phi_{0}\right)
\end{array}\right)\left(\begin{array}{l}
e_{11} \\
e_{22} \\
e_{12}
\end{array}\right) \\
& =\left(\begin{array}{ccc}
\lambda\left(\phi_{0}\right)+2 \mu\left(\phi_{0}\right)-\phi_{0} & \lambda\left(\phi_{0}\right)+\phi_{0} & 0 \\
\lambda\left(\phi_{0}\right)+\phi_{0} & \lambda\left(\phi_{0}\right)+2 \mu\left(\phi_{0}\right)-\phi_{0} & 0 \\
0 & 0 & \mu\left(\phi_{0}\right)-\phi_{0}
\end{array}\right)\left(\begin{array}{l}
e_{11} \\
e_{22} \\
e_{12}
\end{array}\right) .
\end{aligned}
$$

Comparison of (3) and (14) reveals that the effect of applying a uniform hydrostatic stress to an isotropic system is to introduce an explicit pressure dependence in the effective Lame' constants $\lambda^{\prime}$ and $\mu^{\prime}$. This is expected for purely thermodynamic reasons since, for a small strain from the initial state, the initial pressure will do work and thereby change the free energy whose strain derivatives yield the stress-strain relation.

As for systems without initial pressure, further simplifications result if the Cauchy conditions are assumed. Since they arise from the existence of central forces and structural symmetry in the initial state, they have no explicit dependence on an initial hydrostatic pressure. The Cauchy conditions remain as before except for the implicit dependence of the Lame' moduli on initial pressure, i.e. $\lambda\left(\phi_{0}\right)=\mu\left(\phi_{0}\right)$. Calculating the two-dimensional compressibility from (14) for a state of initial hydrostatic pressure $\phi_{0}$,

$$
K_{2 T}\left(\lambda_{2}, \mu_{2}, \phi_{0}\right)=\left[\lambda_{2}\left(\phi_{0}\right)+\mu_{2}\left(\varphi_{0}\right)\right]^{-1}
$$

which may be compared with the corresponding quantity in the bulk for an initial pressure $p_{0}$ :

$$
K_{3 T}\left(\lambda_{3}, \mu_{3}, p_{0}\right)=\left[\lambda_{3}\left(p_{0}\right)+\frac{2}{3} \mu_{3}\left(p_{0}\right)+\frac{1}{3} p_{0}\right]^{-1} .
$$

We conclude from comparison of (15) and (16) that the effect of initial pressure on the compressibility differs in two and three dimensions where explicit pressure dependence appears only in the three-dimensional result. For the case of a Cauchy solid under initial pressure,

$$
K_{2 T}\left(\lambda_{2}, \phi_{0}\right)=\left[2 \lambda_{2}\left(\phi_{0}\right)\right]^{-1}=\left[2 \mu_{2}\left(\phi_{0}\right)\right]^{-1}
$$

and

$$
\begin{aligned}
K_{3 T}\left(\lambda_{3}, p_{0}\right) & =3\left[5 \lambda_{3}\left(p_{0}\right)+p_{0}\right]^{-1} \\
& =3\left[5 \mu_{3}\left(p_{0}\right)+p_{0}\right]^{-1} .
\end{aligned}
$$

Poisson's ratio may also be obtained for simple isotropic (19) or Cauchy isotropic solids (20) subject to initial stress :

$$
\begin{aligned}
\sigma_{2}\left(\lambda_{2}, \mu_{2}, \phi_{0}\right) & =\frac{\lambda_{2}\left(\phi_{0}\right)+\phi_{0}}{\lambda_{2}\left(\varphi_{0}\right)+2 \mu_{2}\left(\phi_{0}\right)-\phi_{0}} \\
\sigma_{2}\left(\lambda_{2}, \phi_{0}\right) & =\frac{1}{3}\left[\frac{1+\phi_{0} / \lambda_{2}\left(\phi_{0}\right)}{1-\phi_{0} / 3 \lambda_{2}\left(\phi_{0}\right)}\right] .
\end{aligned}
$$

These two-dimensional results may be compared with the corresponding bulk expression for isotropic (21) and Cauchy isotropic solids (22) :

$$
\begin{array}{r}
\sigma_{3}\left(\lambda_{3}, \mu_{3}, p_{0}\right)=\frac{\lambda_{3}\left(p_{0}\right)+p_{0}}{2\left[\lambda_{3}\left(p_{0}\right)+\mu_{3}\left(p_{0}\right)\right]} \\
\sigma_{3}\left(\lambda_{3}, p_{0}\right)=\frac{1}{4}\left[1+p_{0} / \lambda_{3}\left(p_{0}\right)\right] .
\end{array}
$$


For the Cauchy solid in two and three dimensions, (20) and (22) may be expressed in more familiar terms through the compressibility from (17) and (18) as

$$
\begin{aligned}
& \sigma_{2}\left(K_{2}, \phi_{0}\right)=\frac{1}{3}\left(\frac{1+2 K_{2} \phi_{0}}{1-\frac{2}{3} K_{2} \phi_{0}}\right) \\
& \sigma_{3}\left(K_{3}, p_{0}\right)=\frac{1}{4}\left(\frac{1+\frac{4}{3} K_{3} p_{0}}{1-\frac{1}{3} K_{3} p_{0}}\right) .
\end{aligned}
$$

Comparison of (23) and (24) reveals that although the effect of initial hydrostatic pressure on the properties of the Cauchy solid is different in two and three dimensions, it is determined in both cases by the dimensionless pressure-compressibility product. This is illustrated in figure 1 where it may be seen that the effect of initial pressure is to increase the value of Poisson's ratio from the zero stress values of $1 / 3$ and $1 / 4$ in two and three dimensions, respectively. We note that the effect of initial pressure is significantly greater for the two-dimensional solid than for the three-dimensional case. In both dimensions, however, the limiting values of 1 and $1 / 2$ for Poisson's ratio in two or three dimensions correspond to fluid-like conservation of area or volume and occur for a pressure-compressibility product of 0.5 . Physically,

or

$$
K_{2} \phi=-(\partial \ln A / \partial \ln \phi)
$$

$$
K_{3} p=-(\partial \ln V / \partial \ln p)
$$

is a dimensionless measure of relative softness for the solid system inasmuch as it is the fractional change in area (volume) per fractional change in pressure required to produce this change. In figure 2 is shown the behaviour of the effective shear modulus $\mu^{\prime}$ as a function of initial pressure. As expected, $u^{\prime}$ vanishes when Poisson's ratio has its maximum value corresponding to fluidlike behaviour. Equivalently, the system does not support transverse sound modes when the pressure-compressibility product reaches its

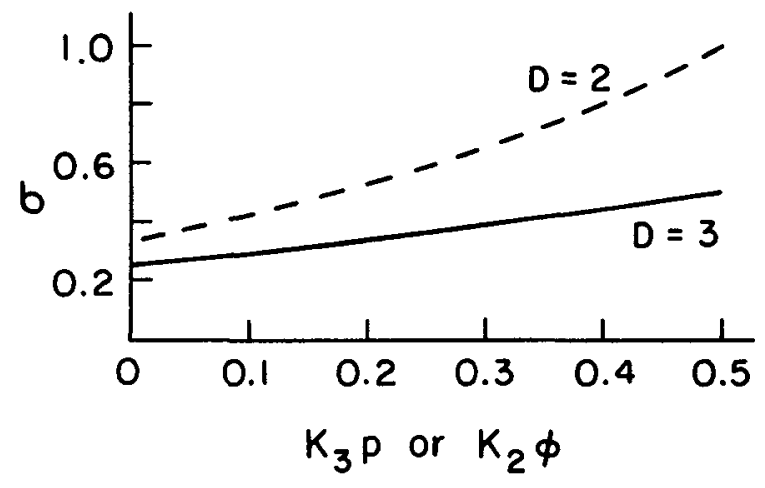

FIG. 1. - Poisson's ratio as a function of relative softness for two and three-dimensional Cauchy solids.

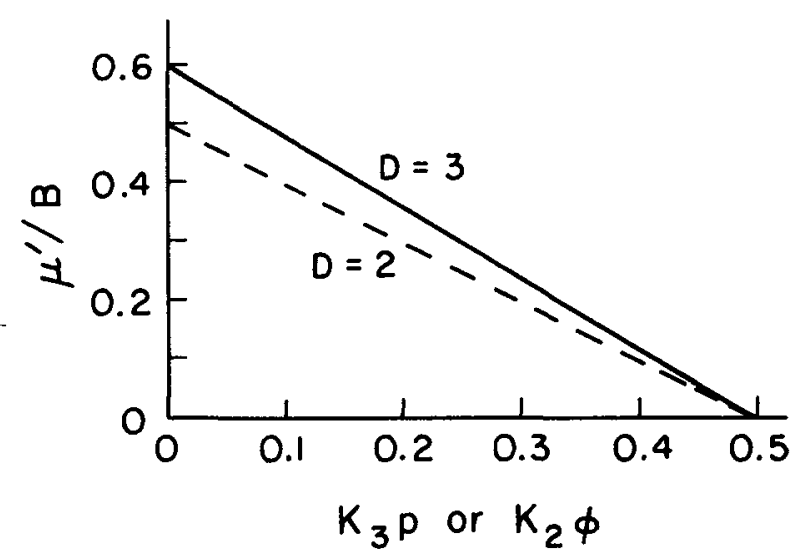

FIG. 2. - The effective shear modulus relative to the bulk modulus $K_{2 T}^{-1}=B$ as a function of relative softness for two and three-dimensional Cauchy solids.

maximum value of 0.5 in two or three dimensions. As indicated in section 3, this general tendency for elastic quantities to depend on the pressure-compressibility product can have a large effect on thermal measurements by significantly altering the zero stress Debye temperatures.

It is interesting that all the information contained in the integration (to obtain $\phi_{0}$ ) and differentiation (to obtain $K_{2}$ ) of the adsorption isotherm is required to write the stress-strain relation for a Cauchy solid when in the general case initial pressure is allowed. This is seen by expressing (14) in terms of the isotherm-derived quantities $\phi_{0}$ and $K_{2}$ :

$$
\left(\begin{array}{l}
\delta T_{11} \\
\delta T_{22} \\
\delta T_{12}
\end{array}\right)=\frac{1}{K_{2}}\left(\begin{array}{ccc}
\frac{3}{2}-\phi_{0} K_{2} & \frac{1}{2}+\phi_{0} K_{2} & \\
\frac{1}{2}+\phi_{0} K_{2} & \frac{3}{2}-\phi_{0} K_{2} & 0 \\
0 & 0 & \frac{1}{2}-\phi_{0} K_{2}
\end{array}\right)\left(\begin{array}{l}
e_{11} \\
e_{22} \\
e_{12}
\end{array}\right)
$$

We conclude that the integration and differentiation of the adsorption isotherm for a two-dimensional solid, equations (1) and (2), is just sufficient to describe the elastic properties of that solid if it is a
Cauchy solid. The implications of this model for heat capacity studies are examined in the following section. 
3. Application of Cauchy solids to heat capacity studies. The previous elastic results may be related to heat capacity measurements in two or three dimensions through the calculation of average sound speeds and thereby the Debye temperature [8]. For two and three-dimensional systems, the Debye temperatures are

$$
\begin{aligned}
& \theta_{2}=\frac{2 \pi \hbar}{k_{\mathrm{B}}}\left(\frac{.1}{\pi a}\right)^{1 / 2} \bar{C}_{2} \\
& \theta_{3}=\frac{2 \pi \hbar}{k_{\mathrm{B}}}\left(\frac{3}{4 \pi v}\right)^{1 / 3} C_{3}
\end{aligned}
$$

where the mean sound speeds in two and three dimensions for isotropic models are given in terms of the transverse and longitudinal speeds by

$$
\begin{aligned}
& 2 \bar{C}_{2}^{-2}=C_{\mathrm{t} 2}^{-2}+C_{12}^{-2} \\
& 3 C_{3}^{-3}=2 C_{t 3}^{-3}+C_{13}^{-3} .
\end{aligned}
$$

These sound speeds may be obtained from the divergence of the stress tensor (14) where

$$
\begin{aligned}
& C_{\mathrm{l}}^{2}=\left(\lambda^{\prime}+2 \mu^{\prime}\right) \rho^{-1} \\
& C_{\mathfrak{\imath}}^{2}=\mu^{\prime} \rho^{-1} .
\end{aligned}
$$

The results (30) and (31) in terms of the effective Lame' constants are the same in two or three dimensions. However, because the functional dependence on $\lambda^{\prime}, \mu^{\prime}$ of alternative elastic variables like the compressibility or Poisson's ratio does depend on dimensionality, the expression for sound speeds in these variables will differ in two and three dimensions. This difference in form also occurs for the Cauchy solid in two and three dimensions where from (14) and the Cauchy expression for the compressibilities is obtained

$$
\begin{aligned}
& C_{12}^{2}=\frac{3}{2}\left(\rho_{2} K_{2}\right)^{-1}\left(1-\frac{2}{3} \phi_{0} K_{2}\right) \\
& C_{\mathrm{t} 2}^{2}=\frac{1}{2}\left(\rho_{2} K_{2}\right)^{-1}\left(1-2 \phi_{0} K_{2}\right)
\end{aligned}
$$

and

$$
\begin{aligned}
& C_{13}^{2}=\frac{9}{5}\left(\rho_{3} K_{3}\right)^{-1}\left(1-\frac{8}{9} p_{0} K_{3}\right) \\
& C_{\mathrm{t} 3}^{2}=\frac{3}{5}\left(\rho_{3} K_{3}\right)^{-1}\left(1-2 p_{0} K_{3}\right) .
\end{aligned}
$$

Comparison of the Cauchy longitudinal or transverse sound speeds reveals a different dependence on initial pressure in two and three dimensions. Using these results the Cauchy Debye temperatures may be calculated in two and three dimensions from (26) and (27),

$$
\begin{gathered}
\theta_{2}=\frac{2 \pi \hbar}{k_{\mathrm{B}}}\left(\frac{2}{\pi a}\right)^{1 / 2}\left(\rho_{2} K_{2}\right)^{1 / 2} f_{2}\left(\phi_{0} K_{2}\right) \\
f_{2}\left(\phi_{0} K_{2}\right)=\frac{1}{4} \sqrt{6}\left[\left(1-2 \phi_{0} K_{2}\right)\left(1-\frac{2}{3} \phi_{0} K_{2}\right) \times\right. \\
\left.\times\left(1-\phi_{0} K_{2}\right)^{-1}\right]^{1 / 2} \\
\phi_{3}=\frac{2 \pi \hbar}{k_{\mathrm{B}}}\left(\frac{9}{4 \pi \mathrm{V}}\right)^{1 / 3}\left(\rho_{3} K_{3}\right)^{1 / 2} f_{3}\left(p_{0} K_{3}\right) \\
f_{3}\left(p_{0} K_{3}\right)=\frac{3 \sqrt{5}}{5}\left(1-\frac{8}{9} p_{0} K_{3}\right)^{1 / 2} \times \\
\times\left[1+6 \sqrt{3}\left(\frac{1-\frac{8}{9} p_{0} K_{3}}{1-2 p_{0} K_{3}}\right)^{3 / 2}\right]^{-1 / 3}
\end{gathered}
$$

where again the dependence on initial pressure as a function of the dimensionless pressure-compressibility product differs in two and three dimensions. The effect of initial pressure on both the sound speeds and Debye temperatures for Cauchy solids is shown in figure 3. In both dimensions the effect of an increasing pressure-compressibility product is to decrease the sound speeds and Debye temperatures, with a given value of the product always having a larger effect for two dimensional solids than for the three

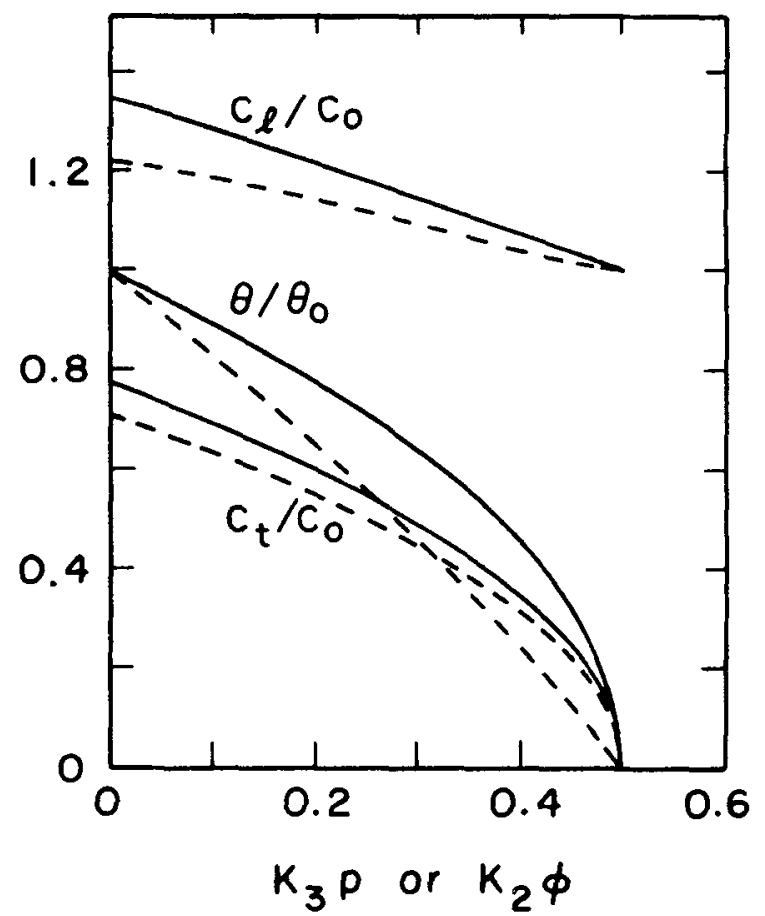

FIG. 3. - Sound speeds and Debye temperatures as a function of relative softness for two (dashed) and three (solid)-dimensional Cauchy solids. The sound speeds are relative to a liquid for which $\rho c_{0}^{2}=K$, and the Debye temperatures are relative to the value $\theta_{0}$ neglecting initial pressure. After ref. [8]. 
dimensional case. As expected, the Debye temperatures vanish for pressure-compressibility products of 0.5 corresponding to the absence of transverse sound modes and fluidlike behaviour.

We now examine if the approximations involved in describing the mechanical properties in terms of a Cauchy solid with initial pressure can describe the Debye temperatures for experimental systems. This has been examined in detail [8] for the case of helium in two and three dimensions where data is available for a wide range of interatomic separations as measured by $v^{1 / 3}$ and $a^{1 / 2}$. Indeed, helium is a useful test system since (a) it is the most compressible of the noble gases and (b) always requires an initial pressure for stability in the solid phase. These two factors provide both a relatively large value for the pressure-compressibility product and a relatively large variation over the total allowed range of 0.5 . As the $0 \mathrm{~K}$ bulk molar volume varies from 13 to $21 \mathrm{~cm}^{3}$, a range of about 700 atmospheres in initial pressure, the relative softness $p K_{3}$ varies from approximately 0.1 to 0.2 . Further, for solid helium there exist direct experimental measurements of the Debye temperatures [10] over this full range in densities. A comparison of predicted bulk $\theta$ 's from the Cauchy solid model and the thermal experimental data of Ahlers is shown in figure 4 . The effect of initial pressure is to decrease the elastic (theoretical) Cauchy $\theta$ 's neglecting pressure into virtual coincidence with the

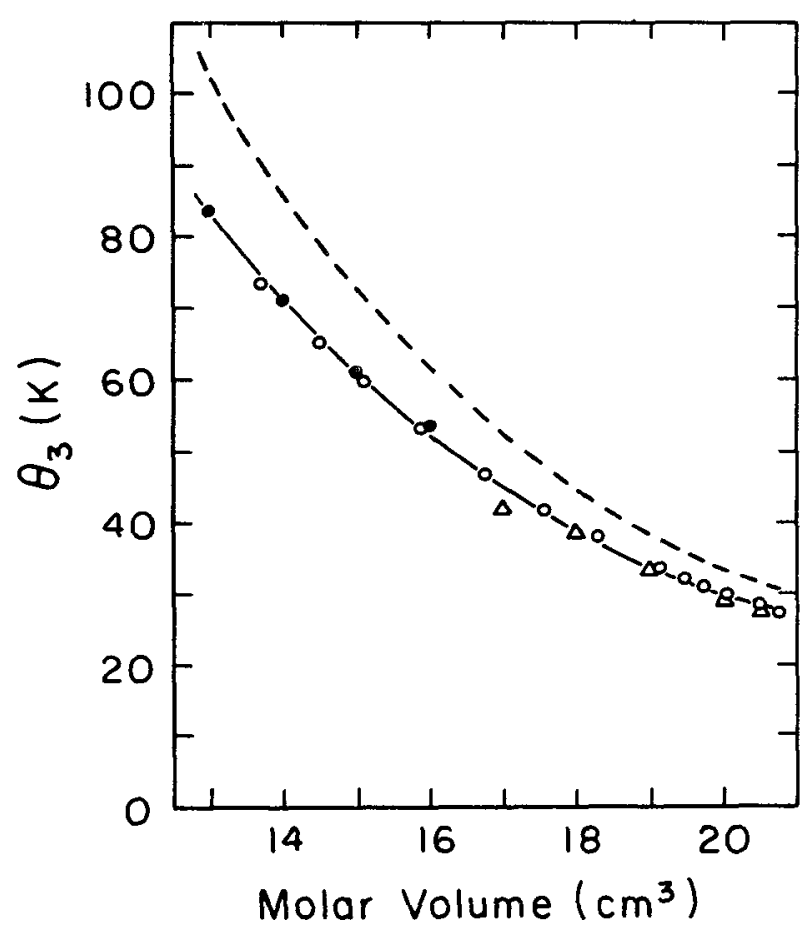

FIG. 4. - Theoretical Cauchy $(0, \Delta)$ and experimental $(0)$ Debye temperatures for bulk helium. The dashed line is the three-dimensional Cauchy solid neglecting initial stress. The experimental points are from ref. [10], the theoretical points and $\Delta$ are calculated from the data of refs. [14] and [15], respectively. After ref. [8]. experimental values. This agreement appears over the full range of molar volumes despite known anisotropies.

Neon is a second system with large compressibilities. Although there exists mechanical isotherm data for neon [11] over a wide range in molar volume, there does not appear to be a correspondingly extensive series of thermal measurements for the Debye $\theta$ 's. However, it is possible to compare approximately the predicted values of Debye temperatures from the Cauchy model for neon if the Debye temperatures at small molar volumes are estimated from the Gruneisen parameter determined at larger molar volumes by Fugate and Swenson [12]. This is shown in figure 5 where the predicted Cauchy values for the bulk Debye temperatures are within $4 \%$ of the low density experimental or high density Gruneisen values for molar volumes ranging from $9 \mathrm{~cm}^{3}$ to the $p=0, T=0$ molar volume of $13.4 \mathrm{~cm}^{3}$. For neon, the bulk values of the pressure-compressibility product are smaller than for helium, ranging from 0.0 to 0.15 where, in contrast to helium, the vanishing lower limit reflects the stable existence of the solid at $T=0$ under zero pressure. Although there is a monotonic deviation of the Cauchy $\theta$ 's from the Debye temperatures obtained from the

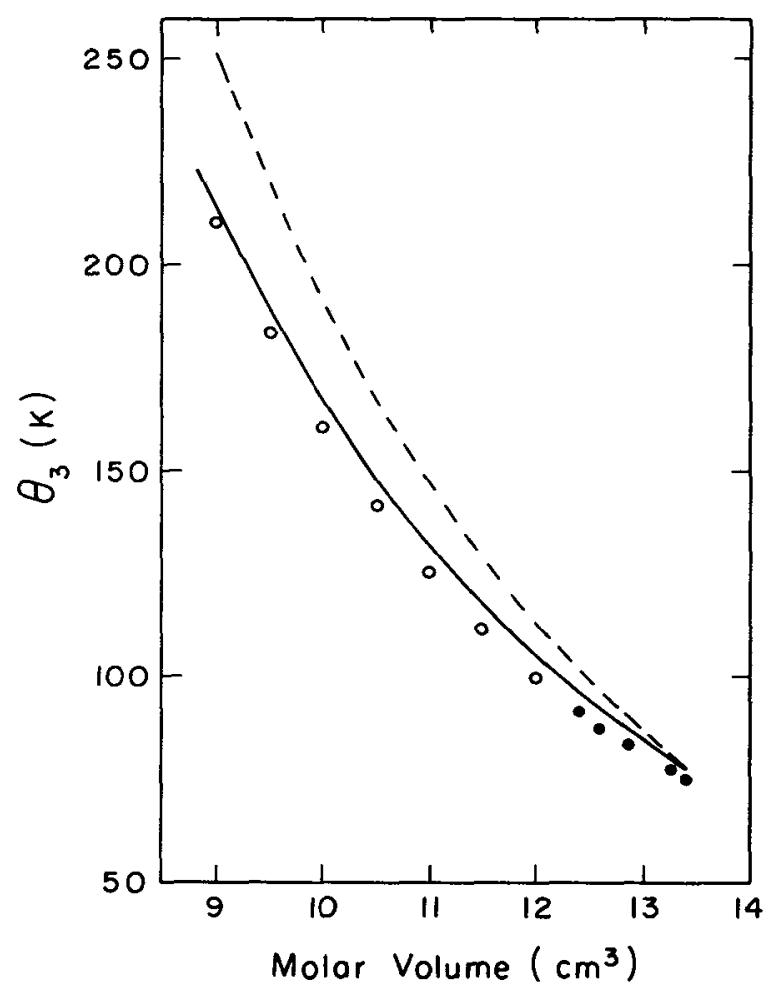

Fro. 5. - Cauchy (dashed or solid line) and experimental (circles) Debye temperatures for bulk neon. The solid circles are experimental data of ref. [12], the open circles are calculated $\theta$ 's using the Gruneisen parameters of ref. [12]. The dashed line neglects initial pressure for the Cauchy solid and the solid line includes initial pressure from the equation of state data of ref. [11]. 
extrapolated experimental values, the variation with pressure is well described by the Cauchy model. Essential agreement is obtained for neon if the Cauchy values are normalized to the $p=0$ experimental value and the pressure variation of the Cauchy model is retained.

The Cauchy solid model may be compared to adsorption systems where there exists compressibility, spreading pressure, and Debye temperature data. Helium remains the only system for which all three quantities are available. Using the spreading pressures and compressibilities deduced from Elgin and Goodstein [13], Cauchy Debye temperatures for two dimensional solids may be generated from (36). The calculated Cauchy $\theta_{2}$ 's and experimental thermal $\theta_{2}$ 's for $\mathrm{He}^{4}$ on Grafoil [1] are shown in figure 6 for the range of atomic areas from (8.9-11.5) $\mathrm{A}^{2}$, corresponding to scaled molar volumes $v \equiv a_{\mathrm{m}}^{3 / 2}$ in the range $(16-23) \mathrm{cm}^{3}$. There is a large pressure induced decrease in the Debye temperatures relative to the values obtained if initial pressure is neglected. This decrease, arising from pressure-compressibility products in the range $0.18-0.21$, varies from $15-25 \%$ of the zero pressure Cauchy values and yields Debye temperatures within $8 \%$ of the experimental values for atomic areas of $10 \mathrm{~A}^{2}$ where the spreading pressure data are most accurate. Agreement is not as good as for the application of the Cauchy solid model to either of the two bulk systems helium or neon. Nevertheless, for monolayer systems as well as the bulk, the effect of initial pressure is significant and must be included with the measured compressibilities in the elastic model if approximate quantitative agreement with the experimental Debye temperatures is to be obtained.

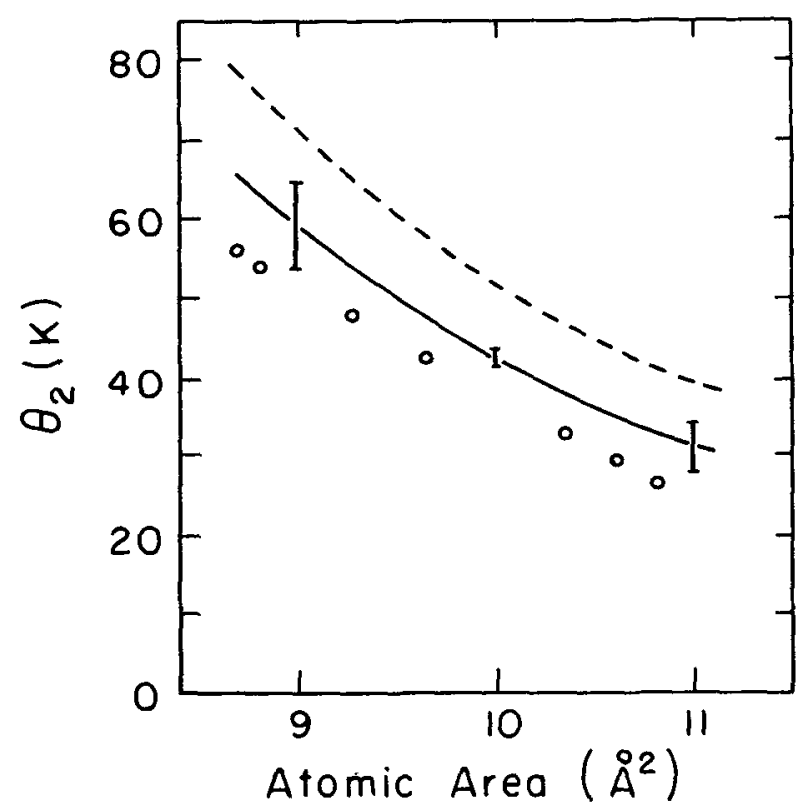

Fig. 6. - Cauchy (dashed or solid lines) and experimental (open circles) Debye temperatures for monolayer helium. The dashed line neglects initial spreading pressure and the solid line includes initial spreading pressure as estimated from ref. [13]. After ref. [8].

We note in conclusion that ordinary transverse and longitudinal sound speed measurements provide a more direct test of elastic models for films than do thermal measurements which involve a combination of the two speeds that arise independently in elastic calculations. Such experimènts would be complicated by the presence of the substrate but, if successful, would yield a shear modulus and hence significantly more information on the elastic properties of monolayer films than is currently available from isotherms and equations (1) and (2).

\section{References}

[1] Bretz, M., Dash, J. G., Hickernell, D. C., MCLean, E. O. and Vilches, O. E., Phys. Rev. A 8 (1973) 1589. [2] Thomy, A. and Duval, X., J. Chem. Phys. 67 (1970) 1101. [3] L,ARHER, Y., J. Chem. Soc. Faraday Trans. 1 (1974) 70, 320

[4] Putnam, F. and Fort, T. Jr., J. Phys. Chem. 79 (1975) 459.

[5] FAIN, S. C. Jr., and ChINN, M. D., and references therein, J. Physique Colloq. 38 (1977) C4-99.

[6] WaLlACE, D. C., Thermodynamics of Crystals (John Wiley \& Sons, Inc., New York), 1972.

[7] Huntington, H. B., in Solid State Physics, Vol. 7 (edited by F. Seitz and D. Turnbull, Academic Press, New York), 1958

[8] Stewart, G. A., Phys. Rev. A 10 (1974) 671.

[9] Drabble, J. R., in The Mechanical Behavior of Materials
Under Pressure (edited by H. L1. D. Pugh, Elsevier, London), 1970.

[10] Ahlers, G., Phys. Rev. A 2 (1970) 1505.

[11] Anderson, M. S., Fugate, R. Q. and Swenson, C. A., $J$ Low Temp. Phys. 10 (1973) 345

[12] Fugate, R. Q. and Swenson, C. A., J. Low Temp. Phys. 10 (1973) 317

[13] Elgin, R. L. and Goodstein, D. L., Phys. Rev. A 9 (1974) 2657.

[14] Dugdale, J. S. and Franck, J. P., Philos. Trans. R. Soc. London A 257 (1964) 1.

[15] Edwards, D. O. and Pandorf, R. C., Phys. Rev. 140 (1965) A816. 